\title{
Basic Steps of Lateral Manipulation of Single Atoms and Diatomic Clusters with a Scanning Tunneling Microscope Tip
}

\author{
L. Bartels, G. Meyer, and K.-H. Rieder \\ FU Berlin, FB Physik, Arnimallee 14, 14195 Berlin, Germany
}

(Received 4 February 1997)

\begin{abstract}
Detailed tip height measurements during manipulation of single atoms, molecules, and dimers on a $\mathrm{Cu}(211)$ surface reveal different manipulation modes depending on tunneling parameters. Both attractive $(\mathrm{Cu}, \mathrm{Pb}, \mathrm{Pb}$ dimers) and repulsive manipulation $(\mathrm{CO})$ are identified. Using attractive forces, discontinuous hopping of $\mathrm{Cu}$ and $\mathrm{Pb}$ atoms from one adsorption site to the next can be induced ("pulling"). $\mathrm{Pb}$ dimers can be pulled with repeated single, double, and triple hops. $\mathrm{Pb}$ atoms can also be "slid" continuously. The occurrence of different movement patterns is shown to be a sensitive probe for surface defects. [S0031-9007(97)03676-4]
\end{abstract}

PACS numbers: 68.35.Fx, 61.16.Ch

The scanning tunneling microscope (STM), invented in 1982 by Binnig and Rohrer [1], was recently further developed from an imaging instrument with atomic resolution to an operative tool [2] with which individual atoms and molecules can be manipulated laterally [3-5] and vertically $[6,7]$ at will. Although different modes of movement of atomic scale have been anticipated for lateral manipulation [3,8-11], no detailed experimental data have been presented up to now. Firm knowledge on the basic mechanisms of single atom manipulation is, however, fundamental for both experimental nanostructuring and theoretical description. The interaction and mobility of adsorbed metal clusters has gained large scientific attention during the last years and is of great technological interest once structures of a few nanometers in size are to be assembled. In this Letter we report on direct experimental evidence of three different kinds of atomic adparticle movement, which can be induced on $\mathrm{Pb}$ atoms, $\mathrm{Cu}$ atoms, and $\mathrm{CO}$ molecules by choosing the appropriate manipulation parameters. Further we applied our experimental method to the smallest possible $\mathrm{Pb}$ clusters and found that we could additionally achieve double and triple adsorption site hops with these dimers. As the dependence of the hop length on the experimental parameters proved to be very minute, we could employ it as an extremely sensitive probe to distant surface defects.

We used a self-built STM [12] at $30 \mathrm{~K}$ to manipulate atoms on the $\mathrm{Cu}(211)$ surface. We used electrochemically etched tungsten tips for all measurements. In situ tip preparation involved mechanical contact between the tip and the $\mathrm{Cu}$ substrate. Hence, the tips used are expected to be covered by $\mathrm{Cu}$ atoms. The following results could be obtained using almost any tip of metallic imaging quality and a resolution sufficient to discern and address single adspecies. Low temperatures are essential for controlled manipulation of small adsorbates to suppress thermal agitation. The adsorption sites of the manipulated adparticles on $\mathrm{Cu}(211)$ as determined previously [5] are indicated in Figs. 1(a) and 1(b). STM imaging is performed with a tun- neling resistance of $\simeq 60 \mathrm{M} \Omega$ and a voltage of $\simeq 50 \mathrm{mV}$. Lateral manipulation involves short range forces between tip and sample [13]. Hence it proceeds by first moving the tip to the desired initial point, then the set point current is increased by 2 orders of magnitude, thus decreasing the tip-sample separation by $\simeq 3.5-4.5 \AA$ (where $\simeq 6.5 \AA$ results in a point contact), and the tip is moved at constant current along the desired line of manipulation whereby the tip height is recorded. Before and after manipulation the contour along the same line is measured at imaging tunneling resistance to determine the initial and final sites of the manipulated species and to rule out uncertainties due to piezo-creep and drift.

The tip height curves during moving of a single $\mathrm{Cu}$ atom, a $\mathrm{Pb}$ atom, a $\mathrm{CO}$ molecule, and a $\mathrm{Pb}$ dimer, respectively, are shown in the left part of Fig. 2. All tip height curves are vertically and horizontally scaled in $\AA$; they are carefully aligned horizontally according to the proper adsorption sites of the manipulated species: The vertical dotted lines indicate the $\mathrm{Cu}$ adatom sites separated by $\vec{a}_{1}$ along [1ํㅣㅇ. On the right part of Fig. 2 surface plots of the different adsorbates are shown. The washboardlike structure surrounding the adsorbates corresponds to the $\mathrm{Cu}(211)$ substrate corrugation. The arrows denote the manipulation direction parallel to the step edges in the surface plane.

Figure 2(a) shows the tip height during moving a $\mathrm{Cu}$ atom. In the initial flat part the tip approaches the $\mathrm{Cu}$ atom. The following sudden upward jump of the tip must be interpreted that the $\mathrm{Cu}$ adatom is attracted by the tip and hops by one adsorption site (distance $\left|\vec{a}_{1}\right|$ ) towards the tip. After this jump the tip moves over the top and then downward along the contour of the $\mathrm{Cu}$ adatom. From the contour line of the $\mathrm{Cu}$ atom we can deduce that its new location is a fcc adatom site again. Once the downward movement of the tip along the side of the atom has reached a threshold depending on the tunneling resistance, another upward jump of the tip occurs [the second in Fig. 2(a)]. At this point the $\mathrm{Cu}$ atom hops by one adsorption site 

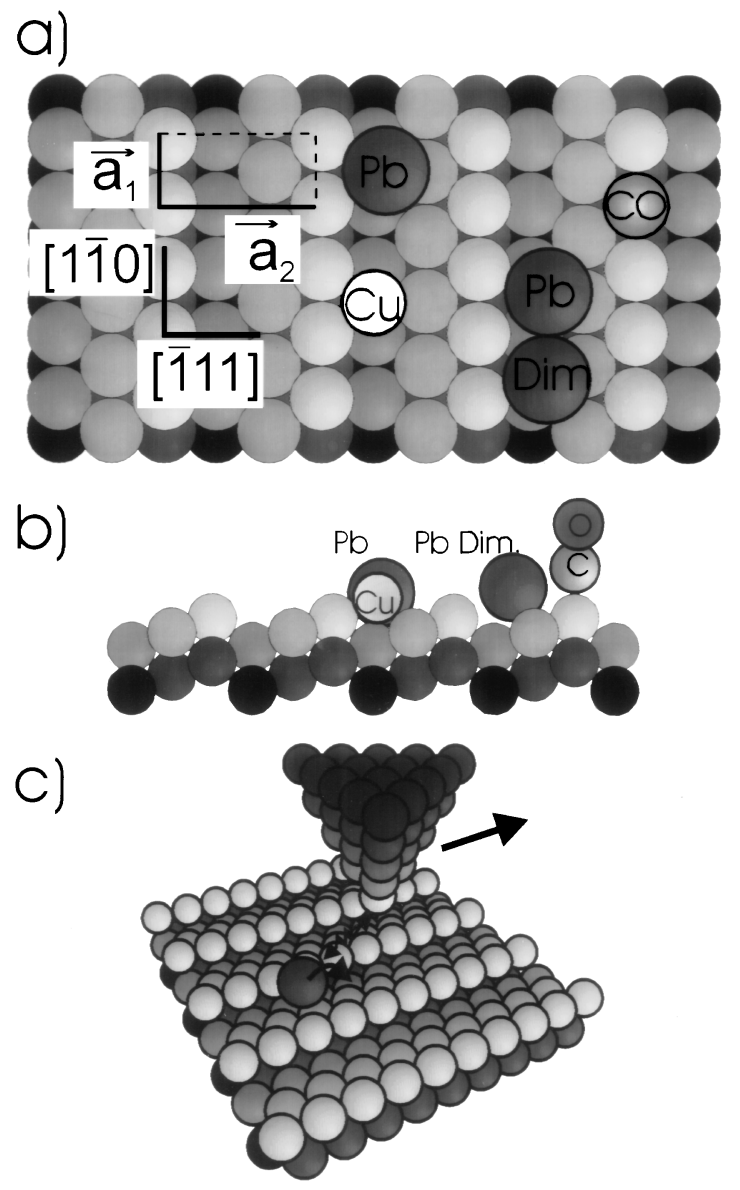

FIG. 1. (a),(b) Top and side views of sphere models of the $\mathrm{Cu}(211)$ surface. The adsorption sites of the manipulated species are indicated. While CO adsorbs on top of the stepedge atoms, $\mathrm{Pb}$ and $\mathrm{Cu}$ atoms occupy fcc sites next to the step edges. The atoms of the $\mathrm{Pb}$ dimer lie near the fcc site but are displaced by $\simeq\left|\vec{a}_{1}\right| / 3$ due to the larger $\mathrm{Pb}$ diameter. In (c) the geometry of the attractive movement of a $\mathrm{Pb}$ atom along the step-edge row with an idealized STM tip is shown.

following the tip. Subsequently the tip moves again over the atom and downward its slope. This process is repeated with the periodicity of the substrate for the rest of the manipulation line. This manipulation mode in which the atom follows the tip discontinuously at varying tip-atom distance we denote as pulling in the following.

The modulation (or jump height) during moving varies for a single tip with gap resistance between 0.3 and $0.8 \AA$ (curve $a$ of Fig. 3) while the total downward displacement of the tip relative to the imaging tip height is in the tunneling resistance regime between 150 and $300 \mathrm{k} \Omega$ a constant of $3.6 \pm 0.1 \AA$ resulting in $\simeq 2.9 \AA$ distance between tip apex and sample surface (curve $c$ of Fig. 3). Nevertheless the lateral displacement of the tip from the center of the manipulated atom immediately before its hop varies between 1.9 and $2.4 \AA$ (curve $b$ of Fig. 3 ). The data set shown in Fig. 3 corresponds to a single tip apex. Different tip apices (or manipulated species)

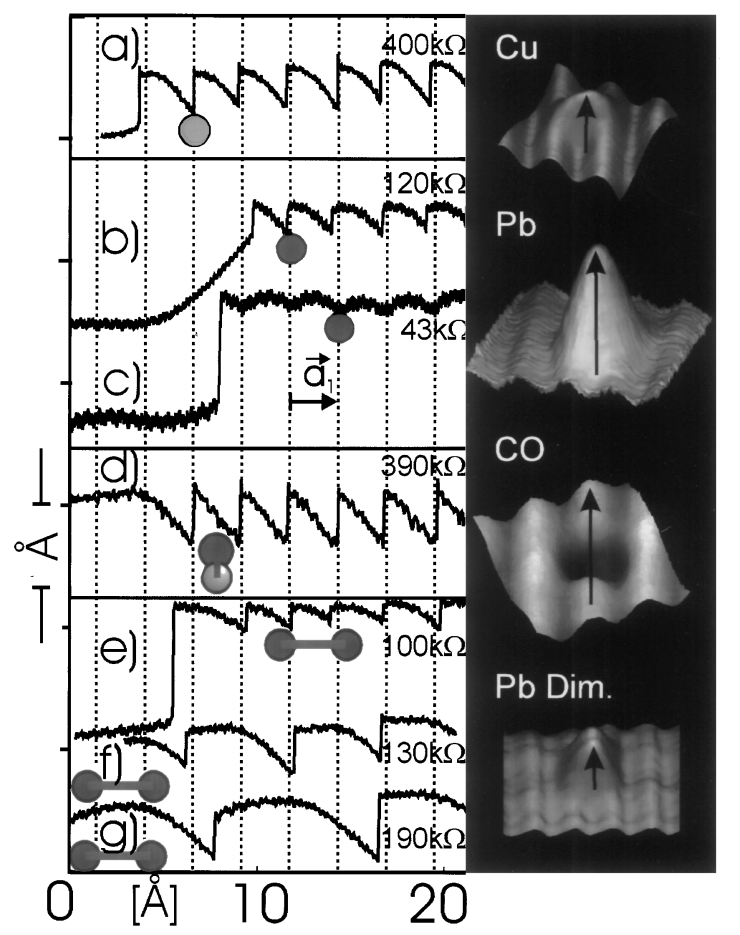

FIG. 2. The left part shows tip height curves during manipulation of a $\mathrm{Cu}$ atom $(a), \mathrm{a} \mathrm{Pb}$ atom $(b, c)$, a $\mathrm{CO}$ molecule $(d)$, and a $\mathrm{Pb}$ dimer $(e)-(g)$ along [1 $\overline{1} 0]$. The tip movement is from left to right, and the tunneling resistances are indicated. All tip height curves are unfiltered raw data. The vertical dotted lines correspond to fcc sites next to the step edge. The initial sites of the manipulated species are indicated by small sphere models. On the right part STM images of the different adparticles are shown. Aspect ratio and angle of view are optimized for each adsorbate. The arrows indicate the direction of tip movement.

show different absolute values but a similar dependence on the gap resistance. The data comprised in Figs. 2 and 3 provide an important basis for detailed theoretical analyses of this basic manipulation mechanism. Verifying the presented data in a simple model based on the undisturbed diffusion barrier along (100) steps on $\mathrm{Cu}(111)$ [14] yields a gradient of the force between tip and adparticle consistent with atomic force measurements by Dürig et al. [13].

Lead atoms adsorb at the same sites as $\mathrm{Cu}$ atoms on $\mathrm{Cu}(211)$ (Fig. 1) but appear significantly larger than $\mathrm{Cu}$ atoms in the STM image (Fig. 2). Nevertheless with regard to manipulation at a tunneling resistance of $120 \mathrm{k} \Omega$ they exhibit the characteristics of pulling: First the tip climbs up the (larger) slope of the $\mathrm{Pb}$ atom until the atom does a single hop towards the tip, indicated by the first upward jump on the measured curve. Then the tip declines along the slope of the atom. Upon reaching a voltage dependent threshold the $\mathrm{Pb}$ atom hops by $\left|\vec{a}_{1}\right|$ following the moving tip and the process starts over again.

Interestingly, decreasing the tunneling resistance to a very low level like $43 \mathrm{k} \Omega$ and thus increasing the tip adparticle force, a different kind of manipulation can be 


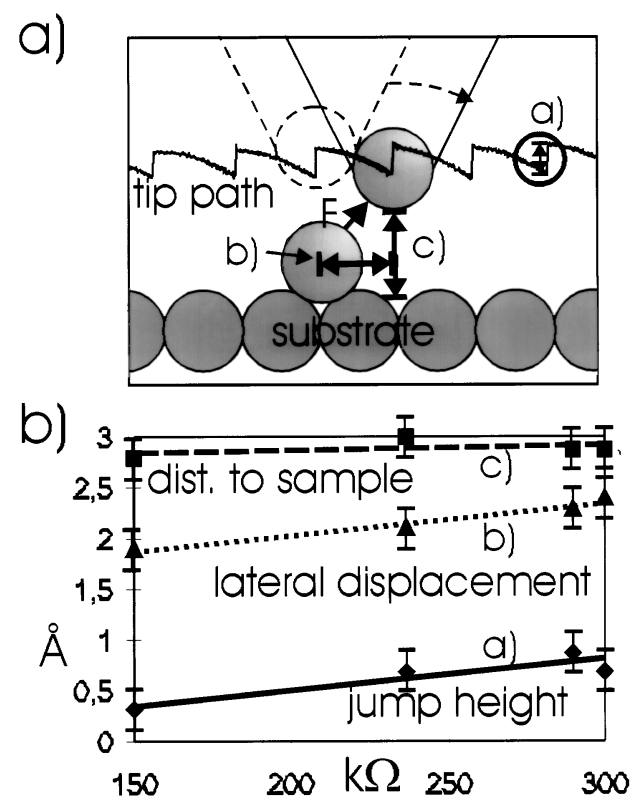

FIG. 3. (a) A measured tip height curve during pulling of a $\mathrm{Cu}$ atom is shown superimposed on a schematic drawing of the positions of the tip apex, the manipulated atom, and the substrate atoms along [1 10$]$. Indicated are the jump height (a), the lateral distance between tip apex and the manipulated $\mathrm{Cu}$ atom at the tip position at which a hop of the $\mathrm{Cu}$ atom occurs $(b)$, and the approximate tip height during manipulation (c) assuming an initial distance of $6.5 \AA$. (b) While the jump height $(a)$ and the lateral distance $(b)$ between tip-apex center and center of the manipulated atom at the point of its hop are strongly dependent on the tunneling resistance, the height of the tip $(c)$ at the point of hopping is less strongly affected. The error bars denote the maximum observed deviation from the indicated means of $0.2 \AA$ in the shown data set corresponding to experiments with a single tip apex.

observed [Fig. 2(c)]. The tip approaches the $\mathrm{Pb}$ atom until it hops towards it by $2.5\left|\vec{a}_{1}\right|$. The tip height curve shows subsequently no discontinuities and its minima correspond to the $\mathrm{Pb}$-adsorption sites. We interpret this as an example of sliding an atom continuously over the substrate. The modulation amplitude during moving the $\mathrm{Pb}$ atom corresponds reasonably well to what would be expected for a $\mathrm{Pb}$ atom gliding on $\mathrm{Cu}(211)$ in a hardsphere model. Retracting the tip after the manipulation stroke leaves in all cases the $\mathrm{Pb}$ atom at the adsorption site on the substrate corresponding to the point of tip withdrawal. Even for this low gap resistance no vertical transfer [6] of $\mathrm{Pb}$ atoms to the tip has been observed.

These two modes of attractively manipulating atoms on a surface might be quite general for lateral manipulation on the atomic scale with the STM tip and are highly likely the ones which have been applied before $[3,15]$. On low index surfaces controlled manipulation of $\mathrm{CO}$ can only be performed using attractive forces. As we use a substrate of high anisotropy, where $\mathrm{CO}$ adsorption is confined to the step edges (Fig. 1), we can also perform controlled repulsive manipulation of $\mathrm{CO}$, without losing the molecules quickly. As an example we present the lateral manipulation of $\mathrm{CO}$ molecules at gap resistances between $\simeq 300$ and $\simeq 600 \mathrm{k} \Omega$. CO molecules on $\mathrm{Cu}(211)$ appear in the STM image in the sombrero shape [15], i.e., as round depression with a protrusion at its center which in the present case (Fig. 2) does not reach the apparent height of the surrounding substrate. Repulsive manipulation of a $\mathrm{CO}$ molecule is shown in Fig. 2(d). In the initial flat part of the curve, the tip approaches the $\mathrm{CO}$ molecule before it starts to descend into the rim of the sombrero until it reaches a threshold depth. Then the tip jumps back to its initial height as the $\mathrm{CO}$ molecule hops by $\left|\vec{a}_{1}\right|$ away from the tip; this makes the tip move up, since it is now outside the apparent depression around the $\mathrm{CO}$ molecule. As the tip moves on it declines again into the depression and the cycle is repeated. On the contour line after the manipulation stroke, the $\mathrm{CO}$ molecule can be found shifted by as many adsorption sites as are jumps in the tip height curve, again supporting pushing mode manipulation.

All lateral manipulations described so far involve continuous movement or hopping of single atoms and small molecules by one adsorption site. Hops by more than one adsorption site can regularly be found for $\mathrm{Pb}$ dimers (the smallest possible $\mathrm{Pb}$ clusters) on $\mathrm{Cu}(211)$. Their hopping range can be tuned to one, two, or three $\left|\vec{a}_{1}\right|$ by the tunneling resistance during manipulation. Employing a relatively low tunneling resistance of $\simeq 100 \mathrm{k} \Omega$ for lateral manipulation results in a force between tip and dimer strong enough that the dimer follows the tip tightly and does single hops [Fig. 2(e)]. Increasing the gap resistance to $\simeq 130 \mathrm{k} \Omega$ makes the dimer perform double hops [Fig. 2(f)] and at a gap resistance of $190 \mathrm{k} \Omega$ the tip moves far down along the slope of the dimer until the in-plane component of the attractive force is strong enough to make the dimer overcome the hopping barrier and to invoke a hop. As the lateral distance between tip apex and dimer center at this point corresponds to more than $2.5\left|\vec{a}_{1}\right|$, once the dimer performs a motion to follow the tip, it does a triple hop [Fig. 2(g)] to reach the energetically most favorable site under the tip apex.

The force needed to manipulate an adparticle depends critically on the direct vicinity of the adparticle and hence can be employed to probe very locally the hopping barrier under presence of the tip. For instance, it is independently of the gap resistance hardly possible to pull a dimer perpendicular to the step edges or to make it pass a $\mathrm{Pb}$ atom incorporated into the nearest step-edge row. Working at a fixed gap resistance the occurrence of single and multiple hops gives us insight into tiny alterations of the hopping barrier. Figure 4 shows an example where a $\mathrm{Pb}$ dimer is passed by a $\mathrm{Pb}$ atom incorporated into the next nearest step-edge row [16] and is then pulled along the edge row of a terrace of the surface. Although no disturbances of the step-edge row, along which the dimer is moved, can be made out in the STM image, 

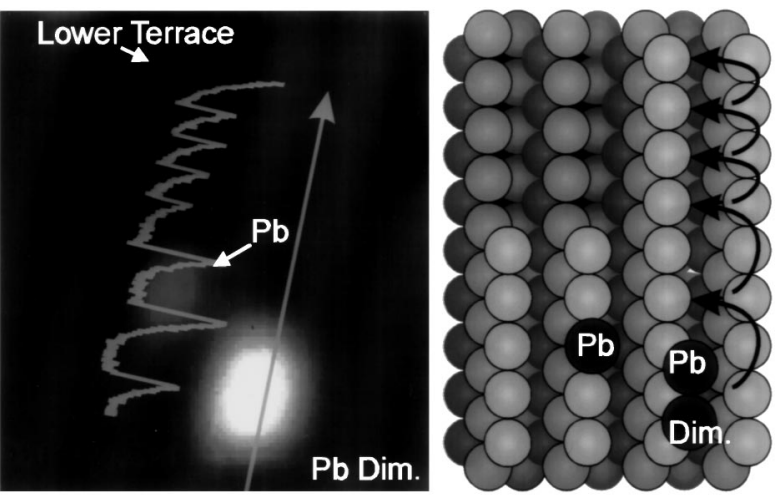

FIG. 4. STM image and hard-sphere model of a $\mathrm{Pb}$ dimer adsorbed next to a step-edge row. In the second step-edge row to the left of the dimer a $\mathrm{Pb}$ atom is incorporated. In the upper part of the image this step-edge row is missing which results in a lower terrace. While manipulating the $\mathrm{Pb}$ dimer along the arrow indicated in the STM image the tip height curve superimposed on the STM image was recorded. In the hard-sphere model the hops of the $\mathrm{Pb}$ dimer corresponding to the central part of the tip height curve are indicated. Passing the $\mathrm{Pb}$ atom in the next-nearest step-edge row double hops corresponding to a comparatively large energetic barrier for hopping were invoked reliably while moving next to the edge of the upper terrace only single hops were produced, i.e., a smaller barrier for tip-induced hopping was seen by the $\mathrm{Pb}$ dimer.

the tip-height curve bears evidence of the mentioned surface defects. When the dimer passes the incorporated $\mathrm{Pb}$ atom double hops are enforced; pulling it along the terrace edge the dimer only performs single hops. As described above, the occurrence of single and multiple hops depends on the delicate balance between the applied force of the tip and the local binding potential of the dimer on the substrate. Especially, the transition from longer to shorter hopping distance is an indication of a decrease in the hopping barrier. As the specific defects causing the reliable transition from double to single hops in this case are more than $8 \AA$ away from the manipulated dimer and the relaxation of an unperturbed $\mathrm{Cu}(211)$ step edge due to an adjacent terrace edge is only of the order of a few $\mathrm{pm}$, these findings are remarkable and bear witness of the extreme sensitivity of this probing method.

In summary, we have shown STM measurements on the behavior of adsorbed atoms and molecules while being laterally manipulated on $\mathrm{Cu}(211)$. Three modes of movement have been demonstrated: Pulling, where the tip proceeds and adparticles like $\mathrm{Cu}$ atoms, $\mathrm{Pb}$ atoms, and $\mathrm{Pb}$ dimers perform hops to follow discontinuously the attractive tip. Sliding of $\mathrm{Pb}$ dimers is observed at lower tunneling resistance where the tip-adsorbate distance remains nearly constant, and a continuous movement of the $\mathrm{Pb}$ atom over the substrate is observed. Pushing, where an adsorbate like $\mathrm{CO}$ is repelled by the approaching tip to do single adsorption site hops away from the tip. More important, we have shown that in the case of simple clusters controlled initiation of hops over multiple adsorption site distances is possible and that the different hopping width can be used in turn to probe effects of adjacent surface defects on the local hopping barrier.

Part of this project was financed by the Deutsche Forschungsgemeinschaft. L.B. wishes to thank the Friedrich-Ebert-Foundation for supporting him with a grant.

[1] G. Binning, H. Rohrer, Ch. Gerber, and E. Weibel, Phys. Rev. Lett. 49, 52 (1982).

[2] Ph. Avouris, Acc. Chem. Res. 18, 95 (1995).

[3] D. Eigler and E. K. Schweizer, Nature (London) 344, 524 (1990).

[4] M. F. Crommie, C. P. Lutz, and D. M. Eigler, Science 262, 218 (1993).

[5] G. Meyer, B. Neu, and K.-H. Rieder, Appl. Phys. A 60, 343 (1995); G. Meyer, S. Zöphel, and K.-H. Rieder, Phys. Rev. Lett. 77, 2113 (1996).

[6] D. M. Eigler, C. P. Lutz, and W.E. Rudge, Nature (London) 352, 600 (1991).

[7] B. Neu, G. Meyer, and K.-H. Rieder, Mod. Phys. Lett. B 9, 963 (1995).

[8] A. Buldum and S. Ciraci, Phys. Rev. B 54, 2175 (1996).

[9] S. Ciraci, E. Tekman, A. Baratoff, and I. Batra, Phys. Rev. B 46, 10411 (1992).

[10] T. A. Jung, R. R. Schlittler, H. Tang, C. Joachim, and J. K. Gimzewski, Science 271, 181 (1996).

[11] P. H. Beton, A. W. Dunn, and P. Moriarty, Appl. Phys. Lett. 67, 1075 (1995).

[12] G. Meyer, Rev. Sci. Instrum. 67, 2960 (1996).

[13] U. Dürig, O. Züger, and D. W. Pohl, Phys. Rev. Lett. 65, 349 (1990).

[14] $228 \mathrm{meV}$ calculated by P. Stoltze, J. Phys. Condens. Matter 6, 9495 (1994); $188 \mathrm{meV}$ measured by G. Schulze Icking-Konert, M. Giese, and H. Ibach (to be published).

[15] J. Stroscio and D. Eigler, Science 254, 1318 (1991).

[16] L. Bartels, S. Zöphel, G. Meyer, E. Henze, and K.-H. Rieder, Surf. Sci. 372, L261 (1997). 